\title{
Are We Solving the Redundancy of Human Motor Control Properly?
}

\author{
Hyungeun Song* \\ Harvard-MIT Division of Health Sciences and Technology, Massachusetts Institute of Technology, USA
}

*Corresponding author: Hyungeun Song, Harvard-MIT Division of Health and Sciences and Technology, Massachusetts Institute of Technology, Cambridge, MA 02139, USA.

To Cite This Article: Hyungeun Song, Are We Solving the Redundancy of Human Motor Control Properly? 2020 - 8(3). AJBSR.MS.ID.001264. DOI: 10.34297/AJBSR.2020.08.001264

Received: 䟧 March 17, 2020; Published: 盋 March 20, 2020

\section{Opinion}

One of the traditional questions in the biomechanics field is identifying human motor control laws. The human motor control system consists of complex biological neural networks and muscles. Yet, it is phenomenal to observe how our motor control can achieve efficient, stable, and robust motion control [1-4]. These intriguing observational results indicate that our central nervous system (CNS) manages to control multiple muscles, which have extremely non-linear dynamics, to drive a multiple linkage structure of the skeletal system to meet biomechanical requirements using limited computational power of CNS and imperfect neural feedback with time delay of peripheral information [5]. Therefore, understanding the principles in human motor control is valuable for not only scientific means but also poses a critical question for implementing affective assistive devices, such as neural prosthesis and exoskeletons, as well as robust autonomous robotics [6,7]. Although various attempts have been made to identify the human motor control law over the past several decades, human motor control is still far from being fully understood. Determining the level of muscle activity for a desired locomotion, also known as load sharing problem (LSP), requires inverse calculation of each muscle activity from given target joint torques or kinematics. Because the nature of the LSP's redundancy due to having greater number of muscles than that of the joints of interest, the analytical solution for the LSP is believed to be unattainable.

\section{Inverse Muscle Activity Estimation Based on Optimality Principle Assumptions}

The standard methodology to deal with the LSP is by assuming the optimality principles in human motor control laws. Each muscle activity is estimated to meet biomechanical requirements such as joint torques and kinematics while optimizing the corresponding cost function for assumed optimality principles. Traditionally, minimization of the total sum of muscle activity [8], muscle endurance [9], or metabolic cost [10] have been used for the optimization scheme. The estimated muscle activity based on the use of optimality principles has provided comparable results to that obtained by electromyography (EMG) of standard movements such as walking, running, and sit-to-stand.

\section{Dynamic Simulation Based on Virtual Limb Model}

The excitation of skeletal muscle to neural signals can be described in first order linear or bi-linear dynamics. To employ these excitation dynamics to an inverse muscle activity estimation, the dynamic simulation methodologies have been proposed [11-13]. The dynamic simulation starts by forward-dynamics simulation of the excitation dynamics and musculoskeletal dynamics. Thereafter, the estimated muscle activity is corrected so that tracking errors between computed joint torques and kinematics by simulated muscle activity and the measured values converge to zeros based on the optimal control theory. The optimality principle in motor control strategy are utilized and, thereby, a cost function is used to determine how each muscle activity is needed to be corrected from joint torque and kinematics tracking errors. Therefore, the dynamic simulation method estimates the muscle activity based on the optimality principles while considering the excitation dynamics. Recently, the dynamic simulation approaches have been widely used for biomechanics studies, evaluation of rehabilitation, and as a simulator for assistive devices.

\section{Are We Solving the Redundancy of Human Motor Control Properly?}

Despite the utilization of the optimality principles in human motor control has been generally accepted by the field, it is worth emphasizing that the demonstration of the neurophysiological terms of the optimality principles are extremely challenging. Conceptually, solving the LSP based on the optimality principle assumption in human motor control is finding an optimal solution 
for the assumed cost function. If the cost function cannot be justified from neurophysiological aspects, the estimated muscle activity is simply one of infinite possible solutions, therefore, their estimation capacity and generality should be questioned. One potential rationale of the field to settle down with these optimality principles-based approaches is because of the analytical solution for the LSP is believed to be unattainable due to its redundancy. However, we may want to step back and defer this conclusion.

\section{State Estimation in Control Theory: Observability}

In the field of control theory, surprisingly, the implementation of analytical inverse estimation of the system states from the system outputs in which the output number is fewer than that of the system states is not new. Although the redundancy between the system states and outputs exists at a given time frame, the analytical solution for the states can be driven based on the system dynamics; the redundancy no longer exists when the scope is extended to the derivatives of states, outputs, and their relationships based on known system dynamics. This estimation principle, also known as system observability, has been first introduced more than six decades ago [14] and has been successfully implemented in several industrial and robotic applications. Furthermore, the advanced state estimation methodologies have been developed for the cases of imperfect system modeling, non-negligible noise, and unknown inputs [15-17]. This implies that if the muscle activity is considered as system states and the joint torque or kinematics is treated as system outputs, the analytical solution can be driven exclusively based on the neuromuscular dynamics while accounting for the unmodeled neural dynamics as system noise. Previous work [18] has shown that the analytical solution can be provided for LSP based on the neuromuscular system observability. To have neurophysiologically unconstrained estimation results of muscle activity, the inverse estimation methodology based on the pure system dynamics needs to be developed rather than simply assuming a cost function to solve LSP.

\section{Is There Redundancy in Human Motor Control?}

System observability argues that the analytical solution exists for LSP exclusively based on the system dynamics for given joint kinematics and torques. One may feel that this is controversial, since our human motor control has extensive degree of freedom (DoF), thereby redundancy exists. The point should be made that the variance in human motor control is observed in a task space, which is described by dimensions including joint kinematics, torque, and impedance, for a given task such as reaching a limb to target points, and walking. Because the DoF of human motor control exceeds that of the task space, the various choices in biomechanical profiles can be made while achieving the same task. This indeed is the redundancy of human motor control, and universal control variables and dimensions that we utilize for motor control have not fully been demonstrated [19-23]. However, this does not necessarily indicate that no analytical solution of muscle activity can be identified from a given specific profile. In other words, the muscle activity estimation, based on the observability, provides the analytical solution exclusively based on known neuromuscular system dynamics for given joint kinematics and torques, but does not attempt to explain why those specific profiles have been produced for given tasks among infinite possibilities [18].

\section{Conclusion}

Load sharing problem (LSP) of the neuromuscular system has been widely discussed for serval decades. However, the development of estimation theory for solving LSP has been held based on the idea that the analytical solution of LSP is unattainable. Meanwhile, we may want to defer this conclusion and explore advanced estimation methodologies such as observability theory to provide a universal solution for LSP, providing better tools for understanding human motor control while considering imperfections of neuromuscular modeling as well as noise in measurements.

\section{References}

1. Kellis E, Arabatz F, Papadopoulos C (2003) Muscle coactivation around the knee in drop jumping using the co-contraction index. J Electromyogr Kinesiol 3(3): 229-238.

2. Nielsen J, Sinkjaer T, Toft E, Kagamihara Y (1994) Segmental reflexes and ankle joint stiffness during co-contraction of antagonistic ankle muscles in man. Exp Brain Res 102(2): 350-358.

3. Jacobs R, Bobbert MF, Van Ingen Schenau GJ (1996) Mechanical output from individual muscles during explosive leg extensions: The role of biarticular muscles. J Biomech 29(4): 513-523.

4. Van Ingen Schenau GJ (1989) From rotation to translation: Constraints on multi-joint movements and the unique action of bi-articular muscles. Human Movement Science 8(4): 301-337.

5. Kandel ER, Schwartz JH, Jessell TM, Siegelbaum SA, Hudspeth AJ (2000) Principles of neural science. ( $5^{\text {th }}$ Edition). McGraw-Hill, New York.

6. Ding Y, Galiana I, Asbeck AT, De Rossi SM, Bae J, et al. (2017) Biomechanical and physiological evaluation of multi-joint assistance with soft exosuits. IEEE Trans Neural Syst Rehabil Eng 25(2): 119-130.

7. Eilenberg MF, Geyer H, Herr H (2010) Control of a powered ankle-foot prosthesis based on a neuromuscular model. IEEE Trans Neural Syst Rehabil Eng 18(2): 164-173.

8. Collins JJ (1995) The redundant nature of locomotor optimization laws. Journal of Biomechanics 28(3): 251-267.

9. Crowninshield RD, Brand RA (1981) A physiologically based criterion of muscle force prediction in locomotion. J Biomech 14(11): 793-801.

10. Miller RH, Umberger BR, Hamill J, Caldwell GE (2012) Evaluation of the minimum energy hypothesis and other potential optimality criteria for human running. Proc Biol Sci 279(1733): 1498-1505

11. Hussain S, Xie SQ Jamwal PK (2013) Effect of cadence regulation on muscle activation patterns during robot-assisted gait: A dynamic simulation study. IEEE J Biomed Health Inform 17(2): 442-451.

12. Shourijeh MS, Smale KB, Potvin BM, Benoit DL (2016) A forwardmuscular inverse-skeletal dynamics framework for human musculoskeletal simulations. Journal of Biomechanics 49(9): 17181723.

13. Thelen DG, Anderson FC (2006) Using computed muscle control to generate forward dynamic simulations of human walking from experimental data. J Biomech 39(6): 1107-1115.

14. Kalman RE (1960) On the general theory of control systems. IFAC Proceedings 1(1): 491-502. 
15. Simon D (2006) Optimal state estimation: Kalman, H infinity, and nonlinear approaches. Hoboken, NJ: John Wiley \&Sons.

16. Chen WH, Yang J, Guo L, Li S (2016) Disturbance-observer-based control and related methods-An overview. IEEE Transactions on Industrial Electronics 63(2): 1083-1095.

17. Guan Y, Saif M (1991) A novel approach to the design of unknown input observers. IEEE Transactions on Automatic Control 36(5): 632-635.

18. Song H, Hori Y (2019) Evaluation of Lower Limb Neuromuscular System Observability and Estimability of Muscle Activity. J Mot Behav pp.1-17.

19. Scholz JP, Schöner G (1999) The uncontrolled manifold concept: identifying control variables for a functional task. Experimental brain research 126(3): 289-306.
20. Papi E, Rowe PJ, Pomeroy VM (2015) Analysis of gait within the uncontrolled manifold hypothesis: stabilisation of the centre of mass during gait. J Biomech 48(2): 324-331.

21. Monaco V, Tropea P, Rinaldi LA, Micera S (2018) Uncontrolled manifold hypothesis: organization of leg joint variance in humans while walking in a wide range of speeds. Hum Mov Sci 57: 227-235.

22. Steele KM, Rozumalski A, Schwartz MH (2015) Muscle synergies and complexity of neuromuscular control during gait in cerebral palsy. Dev Med Child Neurol 57(12): 1176-1182.

23. Hagio S, Fukuda M, Kouzaki M (2015) Identification of muscle synergies associated with gait transition in humans. Front Hum Neurosci 9: 48 